\title{
Virus EB, transplantation hépatique, et syndrome lymphoprolifératif post-transplantation
}

Un syndrome lymphoprolifératif (SLP) post-transplantation - polyclonal ou monoclonal, diffus ou focal, aigu ou insidieux - survient chez 1 à $10 \%$ des transplantés [1]. La présence quasi constante du virus EB y est bien établie, répondant primitivement à un état d'infection latente de cellules lymphoïdes B au sein du SLP [2]. Celui-ci, associé au traitement immunosuppresscur agressif posttransplantation, relève donc très probablement d'une réactivation de l'infection $\mathrm{EB}$ latente d'un petit nombre de cellules $B$ à croissance jusque-là contrôlée - une infection première en période posttransplantation augmentant, bien entendu, les risques de lymphome. Ces cellules lymphoïdes infectées hébergent les ćpisomes du virus $\mathrm{EB}$, formes circulantes du génome viral trouvé dans les infections latentes; ensuite, l'expression de trois des gènes du répertoirc limité exprimé par les épisomes mène la prolifération virale [3]. L'expression du gène EBER-1, codant pour un petit ARN messager, est également observée tôt au cours de l'infection EB latente; bien que les transcrits de ces gènes, dont la fonction est inconnue, ne soient pas nécessaires à la prolifération lymphoïde, leur expression est forte - jusqu'à $10^{7}$ copies par cellule - au cours de l'infection latente par le virus EB [4]. L'intérêt de la détection de l'ARN EBER-1 pour identifier les cellules infectées par le virus EB sur des tissus fixćs par le formol et en coupes incluses dans la paraffine a été démontré [5].

C'est sur ces bases que Randhawa $e t$ al. [6] (division d'anatomie pathologique de la transplantation, Pittsburgh, USA) ont étudié par hybridation in situ l'expression du gène EBER-1 sur des biopsies hépatiques d'enfants et de jeunes adultes ayant eu une allogreffe de foie, et ayant ou n'ayant pas développé un SLP post-transplantation. Le pre-
SLP dans un délai de 5 à 640 semaines $(12,5$ semaines en moyenne) post-greffe ; la première biopsie hépatique a été faite deux jours à 22 mois avant l'éclosion du SLP. La localisation de celui-ci, lors du diagnostic, était le foie (7 cas), les amygdales ou le tissu lymphoïde du cavum (5 cas), le tube digestif, la trachée, les voies biliaires, les méninges ou les ganglions périphériques dans cinq cas, et était d'emblée disséminée dans trois cas. Dans le groupe témoin de 20 enfants (10 mois à 16 ans) n'ayant pas développé de SLP (biopsie hépatique 5 à 1790 jours après la transplantation, surveillance 9 à 71 mois après la biopsie, soit au total jusqu'à 539 semaines après transplantation), le spectre clinique des maladies ayant conduit à la transplantation hépatique était le même que dans le groupe SLP. Soixante et onze pour cent (17 sur 24) des sujets développant un SLP avaient de 1 à $40 \%$ de cellules mononucléées hépatiques positives pour le gène EBER-1, $59 \%$ (10 sur 17) de ceux-ci ayant une histopathologie évocatrice d'hépatite à virus $\mathrm{EB}$. En revanche, $10 \%$ (2 sur 20) seulement des sujets du groupe témoin avaient de rares cellules EBER-1 positives. L'intérêt majeur de ce travail est que l'expression d'EBER-1 a été trouvée 17 fois sur 24 avant (2 jours à 22 mois) le développement du SLP. L'intervalle entre la détection de l'ARNm EBER-1 et l'éclosion de celui-ci était de 0 à 50 jours chez 10 malades, de 51 à 100 jours chez trois malades et supérieur à 100 jours chez quatre malades. Chez 10 des 17 sujets où des cellules EBER-1 positives ont été identifiées dans le foie, le lymphome ultérieur s'est lui aussi développé dans le foie, et dans les sept autres cas dans les organes extra-hépatiques. L'expression du gène EBER -1 a été démontrée dans les 11 tissus atteints par le SLP qui ont pu être, eux-mêmes, étudiés par hybri- dation in situ. L'ARNm de l'EBER-1 a, non seulement, été détecté dans des cellules mononucléées du foie, mais aussi dans un petit nombre d'hépatocytes, constatation inattenduc puisqu'on pensait que le virus EB n'infectait pas d'autres cellules que les cellules lymphoïdes et les cellules épithéliales des muqueuses : il est possible que la positivité EBV des hépatocytes soit le résultat d'une fusion entre hépatocytes et lymphocytes infectés, le virus EB étant un promoteur de fusion cellulaire. Les cellules épithéliales des ductules biliaires et les cellules endothéliales étaient toujours négatives pour l'ARNm EBER-1. La majorité des cellules lymphoïdes positives étaient $\mathrm{CD}_{20}{ }^{+}$, très peu étaient $\mathrm{CD} 43^{+}$, ce qui suggère que les cellules EBER-1 étaient surtout $B$. Tous les sujets séropositifs pour l'EBV avaient de l'ARNm EBER-1 dans leur foie, alors qu'inversement six patients sćronégatifs (et développant un SLP) étaient négatifs pour l'ARNm EBER-1.

Finalement, ce travail montre : (a) que les lymphocytes des sinusoïdes et des espaces portes hépatiques pcuvent abriter le virus EB, comme peuvent le faire la muqueuse oro-pharyngée, la glande lacrymale, les ganglions lymphatiques ou les lymphocytes circulants ; et surtout que (b) cette infection peut précéder l'éclosion clinique et histopathologique évidente d'un SLP post-transplantation hépatique. La valeur prédictive, à l'échelon individuel, de la positivité EBER-1 en matière de lymphome post-greffe mériterait d'être confirmée par des études prospectives : mais la détection de telles cellules positives en l'absence de signes de rejet devrait inciter le clinicien à diminuer significativement l'immunosuppression, puisque l'infection par le virus $\mathrm{EB}$ répond, même en cas de syndrome prolifératif avancé, à l'immunomodulation. Est intéressant à noter le fait que la présence de cellu- 
les lymphoïdes positives pour le virus $\mathrm{EB}$, précédant la survenue d'un SLP, a également été signalće dans les ganglions lymphatiques de sujets atteints de SIDA [7], notion s'accordant avec le processus de carcinogenèse à étapes induit par ce virus. Contrairement à celles établies dans les carcinomes nasopharyngiens [8], la responsabilité directe du virus EB dans la survenue des lymphomes post-transplantation n'est pas encore formellement démontrée, mais il est de plus en plus clair, à la lumière de travaux comme celuici, qu'il y jouc un rôle essentiel.

C. M.

1. Hanto DW, Gajl-Peczalska $\mathrm{KJ}$, Frizzera $\mathrm{G}$, et al. Epstein-Barr virus (EBV) induced polyclonal and monoclonal B-cell lymphoproliferative diseases occurring after renal transplantation : clinical, pathologic, and virologic findings and implications for therapy. Ann Surg 1983 ; 198 : 356-69. 2. Young I, Alfieri C, Hennessy $\mathrm{K}$, et al. Expression of Epstein-Barr virus transformationassociated genes in tissues of patients with EBV lymphoproliferative disease. $N$ Engl J Med 1989 ; $321: 1080-5$.

3. Kieff E, Liebowitz D. Epstein-Barr virus and its replication. In : Fields BN, Knipe DM, eds. Fields Virology, 2nd ed, vol. 2. New York; Raven Press, 1990 ; 1889-920.

4. Rooney C, Howe JG, Speck SH, Miller G. Influences of Burkitt's lymphoma and primary $\mathrm{B}$ cells on latent gene expression by the nonimmortalizing P3J-HR-1 strain of Epstein-Barr virus. $J$ Virol 1989; $63: 1531-9$.

5. Weiss LM, Chenn YY, Liu XF, Shibata D.

Epstein-Barr virus and Hodgkin's disease : a correlative in situ hybridization and polymerase chain reaction study. Am J Pathol 1991 ; 139 : 1259-65.

6. Randhawa PS, Jaffre R, Demetris AJ, et al. Expression of Epstein-Barr-virus-encoded small RNA (by the EBER-1 gene) in liver specimens from transplant recipients with post-hepatic transplantation lymphoproliferative disease. $N$ Engl J Med $1992 ; 327: 1717-4$.

7. Shibata D, Weiss LM, Nathwani BN, Brynes RK, Levine AM. Epstein-Barr virus in benign lymph node biopsies from individuals infected with the human immunodeficiency virus is associated with concurrent or subsequent development of non-Hodgkin's lymphoma. Blood $1991 ; 77: 1527-3$.

8. Raab-Traub N. Epstein-Barr virus and nasopharyngeal carcinoma. Semin Cancer Biol 1992 ; 3 : 297-307. 